\title{
METÁSTASIS RENAL DE HEPATOCARCINOMA
}

\author{
E. SANZ MAYAYO, T. MAYAYO DEHESA, I. GÓMEZ GARCÍA, J. SÁENZ MEDINA, \\ R. RODRÍGUEZ-PATRÓN RODRÍGUEZ, A. ESCUDERO BARRILERO
}

Servicio de Urología. Hospital Ramón y Cajal. Madrid.

Actas Urol Esp. 27 (5): 387-390, 2003

\section{RESUMEN}

"METÁSTASIS RENAL DE HEPATOCARCINOMA"

OBJETIVO: Presentar el caso de una metástasis renal solitaria secundaria a hepatocarcinoma.

MÉTODO: Describimos el caso de un paciente de 51 años que en ecografía abdominal se descubre incidentalmente una masa renal izquierda y una masa hepática. En el TAC se objetivó la masa renal izquierda de $17 \mathrm{~cm}$ de diámetro con posible afectación de vena renal izquierda y una masa hepática en lóbulo derecho. La PAAF guiada por TAC de ambas masas demostró en hígado celularidad maligna compatible con hepatocarcinoma, y en riñón celularidad maligna compatible con carcinoma renal o suprarrenal. Se indicó tratamiento quirúrgico con nefrectomía radical izquierda más hepatectomía derecha.

RESULTADO: El análisis histopatológico de la pieza mostró hepatocarcinoma trabecular moderadamente diferenciado con metástasis ganglionar y renal izquierda.

PALABRAS CLAVE: Metástasis renal. Hepatocarcinoma.

\section{ABSTRACT \\ "RENAL METASTASIS OF HEPATOCELLULAR CARCINOMA"}

OBJECTIVE: To report the case of a solitary renal metastasis secondary to hepatocellular carcinoma.

METHODS: We report the case of a 51 year old patient who on abdominal ultrasonography was revealed a left renal tumour and a hepatic mass incidentally. A TAC showed the left renal tumor measuring $17 \mathrm{~cm}$ in size, possible involvement of left renal vein and a tumour mass in the right lobe of the liver. A TAC guided fine needle punction aspiration biopsy demonstrated a malignant hepatic lesion compatible with hepatocarcinoma, and malignant renal cells compatible with renal or adrenal carcinoma. Left radical nephrectomy and right hepatectomy was performed.

RESULTS: Histopathologic study confirmed the diagnosis of moderately differentiated trabecular hepatocarcinoma with lymph node and left renal metastasis.

KEY WORDS: Hepatocellular carcinoma. Renal metastasis.

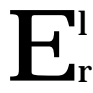
descubrimiento sincrónico de una masa renal izquierda y una masa hepática constituye un fenómeno poco frecuente. Se plantea una duda diagnóstica sobre el origen, pudiendo ser éste único, renal o hepático, o bien ser ambos tumores primarios. Presentamos un caso de estas características, que inicialmente sugería, por el aspecto radiológico, la presencia de un adenocarcinoma renal con metástasis hepática.

\section{CASO CLÍNICO}

Paciente varón de 51 años, con antecedentes de VHC positivo sin hepatopatía clínica, que en ecografía abdominal de control se encuentra 
como hallazgo incidental una masa renal izquierda de 136 por $112 \mathrm{~mm}$ sólida y heterogénea y una lesión hepática de 72 por $39 \mathrm{~mm}$ (Fig. 1).

El paciente no tenía ningún tipo de sintomatología acompañante. A la exploración se palpa una masa sólida en flanco izquierdo no adherida a planos y una discreta hepatomegalia.

En las determinaciones analíticas sólo es reseñable cifras de AST de 75 UI, ALT de 48 UI y GGT de 78 UI.

Ante este hallazgo se solicitó la realización de una TAC que mostró una masa renal izquierda de 17 centímetros de diámetro, sólida, sin áreas necróticas, que se acompaña de una adenopatía aislada y con posible afectación de vena renal izquierda. También se objetivó una metástasis hepática en lóbulo derecho (Figs. 2 y 3).

Para completar el estudio de extensión se realizó gammagrafía ósea y TAC cerebral que no mostraron alteraciones.

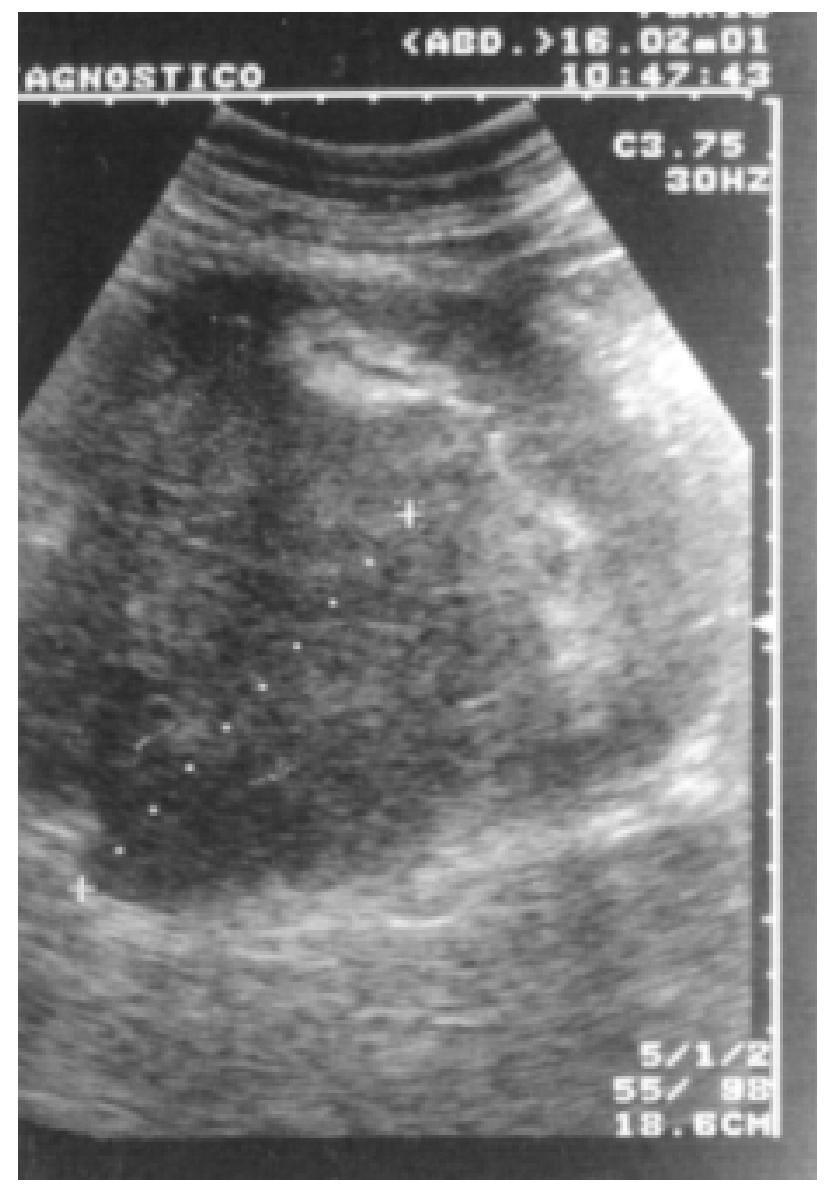

FIGURA 1. Ecografia: masa renal izquierda de 136 por $112 \mathrm{~mm}$ sólida y heterogénea.

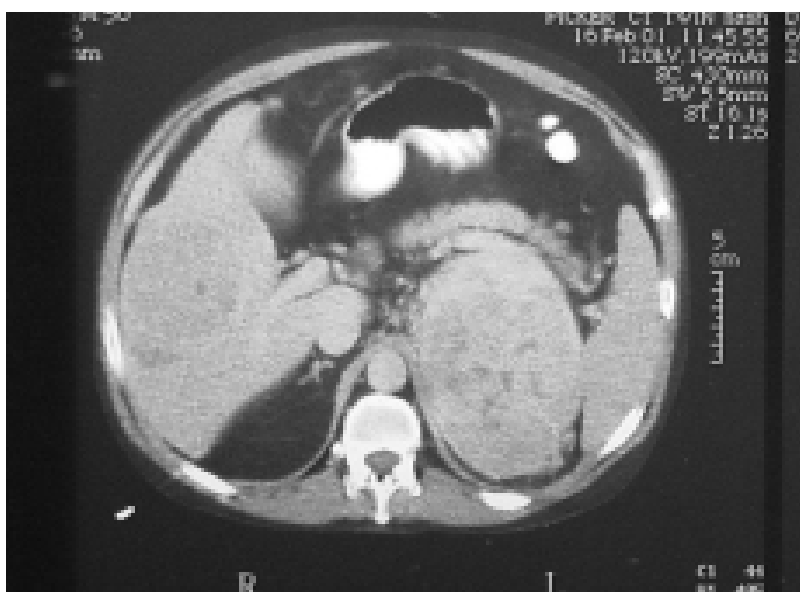

FIGURA 2. TAC: tumoración renal izquierda solida acompañada de tumoración hepática en lóbulo derecho.

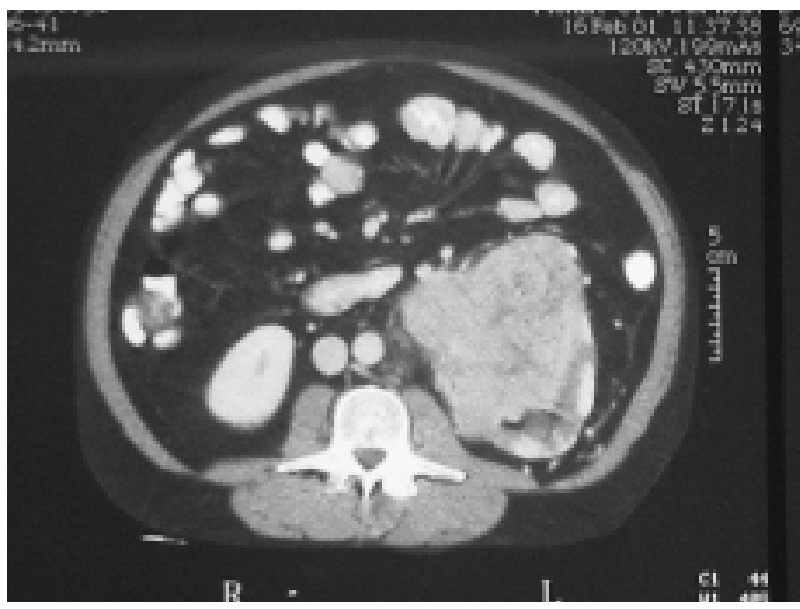

FIGURA 3. TAC: tumoración sólida a nivel de riñón izquierdo de $17 \mathrm{~cm}$ de diámetro.

Se realizó también angiorresonancia objetivando masa renal izquierda de tercio mediosuperior, que en su crecimiento engloba a la arteria renal izquierda desplazando en sentido anterior a la vena renal del mismo lado. Adenopatías en hilio renal izquierdo, la mayor de unos dos centímetros. En lóbulo derecho hepático se observan tres masas de cinco, cuatro y ocho centímetros.

Ante estos hallazgos se pensó como opción más probable en un tumor renal primario con metástasis hepáticas, para confirmarlo se realizó punción aspiración bajo control radiológico con TAC. El estudio citológico demostró en hígado celularidad maligna compatible con hepatocarcinoma y en riñón celularidad maligna compatible con carcinoma renal o suprarrenal. 
Con el diagnóstico preoperatorio de adenocarcinoma renal y carcinoma hepático, se decide tratamiento quirúrgico. Durante la intervención se halla tumoración renal izquierda de $17 \mathrm{~cm}$ de diámetro con compresión de arteria renal izquierda y dos tumores hepáticos de $6 \mathrm{~cm}$ de diámetro localizados en segmento V, VI y VII. Se realiza nefrectomía radical izquierda, linfadenectomía retroperitoneal y hepatectomía derecha.

Se envió una cuña de tejido hepático para estudio histopatológico intraoperatorio que mostró signos de hepatopatía crónica por VHC.

El paciente cursó con una buena recuperación post-operatoria extubándose a las 72 horas y recuperando la función hepática.

A los diez días de la cirugía el paciente reingresó en la UCI por un episodio de hemorragia digestiva alta que se complicó con un fracaso renal agudo secundario a bajo gasto, encefalopatía hepática y sepsis. Se descubrió una peritonitis bacteriana por Enterobacter.

Presentó episodios de ascitis recidivante tras punción evacuadora que provocó una insuficiencia respiratoria secundaria por la compresión.

Se le realizó TAC toraco-abdominal que objetivó derrame pleural bilateral y dos nódulos pulmonares en lóbulo superior e inferior derechos de 1 a $2 \mathrm{~cm}$ (no presentes en TAC previo). Ascitis con colecciones intra y retroperitoneales y signos de hipertensión pulmonar.

El paciente evolucionó desfavorablemente falleciendo a los 45 días de la cirugía.

El estudio histopatológico de la pieza mostró hepatocarcinoma trabecular moderadamente diferenciado con metástasis ganglionares y renal izquierda de gran tamaño.

\section{DISCUSIÓN}

Aproximadamente el 90\% de los hepatocarcinomas celulares asientan sobre una hepatopatía crónica, como sucede en este caso. Las metástasis extrahepáticas clínicamente manifiestas son muy poco frecuentes, aunque en las necropsias se pueden encontrar en un número importante de $\operatorname{casos}^{1}$.

Las localizaciones más frecuentes de metástasis del hepatocarcinoma son: ganglios linfáticos regionales, pulmón, hueso, glándula adrenal y cerebro ${ }^{2}$.
En un estudio realizado por Nakashima et al. en pacientes fallecidos por hepatocarcinoma se descubrió que de 225 en 144 existían metástasis extrahepáticas (64\%), y en 5 metástasis renales $(2,2 \%)^{3}$.

El riñón, dada su rica vascularización, es un lugar frecuente para albergar metástasis de diversos tumores sólidos y neoplasias hematológicas. Los tumores que más frecuentemente metastatizan en el parénquima renal son los de pulmón y mama, más raramente el coriocarcinoma ${ }^{4}$. Las metástasis no suelen ser detectadas clínicamente $\mathrm{y}$ se suelen descubrir en las necropsias ${ }^{5}$, esto se debe probablemente a que los pacientes son portadores de tumores muy avanzados y no viven lo suficiente para que la lesión renal ocasione sintomatologia ${ }^{4}$.

En ocasiones pueden producir síntomas como dolor lumbar, hematuria o incluso se han descrito casos de hemorragias masivas al espacio retroperitoneal ${ }^{6}$.

Las metástasis en riñón suelen ser múltiples y de pequeño tamaño aunque en ocasiones pueden presentarse como infiltración difusa o nódulos únicos. La peculiaridad de este caso viene dada por el importante volumen del nódulo metastático, que nos hizo pensar como primera posibilidad en un tumor primario renal.

Las metástasis renales del hepatocarcinoma clínicamente manifiestas son excepcionales, en la revisión bibliográfica hemos encontrado cinco casos de estas características ${ }^{6-9}$.

Ni con la ecografía, ni con el TAC o RNM es posible diferenciar un tumor primario renal de una metástasis por lo que se precisa la realización de una punción aspirativa con aguja fina para su correcta identificación ${ }^{10}$. En este caso ni siquiera con ésta se pudo alcanzar el diagnóstico.

El único tratamiento eficaz en este tipo de pacientes es el quirúrgico, teniendo en cuenta que hay que hacer una correcta selección de los mismos dada la elevada morbi-mortalidad de esta cirugía $^{11}$. En el presente caso dada la edad del paciente y la ausencia de factores de riesgo, se planteó en una sesión única, comenzando con la nefrectomía y linfadenectomía y posteriormente la hepatectomía parcial. 


\section{REFERENCIAS}

1. LEE YM, GEER DA.: Primary liver cancer: pattern of metastasis. J Surg Oncol 1987; 36: 26.

2. HA-KAWA SK, HARIMA K, TANAKA Y et al.: A clinical evaluation of distant metastases from hepatocellular carcinoma with reference to location, frequency, symptoms, treatment and prognosis. Gan No Rinsho 1990; 36: 2012.

3. NAKASHIMA T, OKUDA K, KOJIRO M et al.: Pathology of hepatocellular carcinoma in Japan. 232 consecutive cases autopsied in ten years. Cancer 1983; 51: 863.

4. ESCUDERO A.: Metástasis en el riñón secundarias a tumores primitivos de otros órganos. En: adenocarcinoma renal. Editorial AC. Madrid 1980: 119-122.

5. CAROLYN M, DRESLER MD.: Kidney metastases. Chest Surgery Clinics of North America 1998; 8: 127.

6. MEZAWA S et al.: Spontaneous rupture of renal metastasis of hepatocellular carcinoma: management by emergency transcatheter arterial embolization. Cardivasc Intervent Radiol 2001; 24: 143.

7. HSU YB, LEE PH, SEP JC, CHEN DS, HSU HC.: Hepatocellular carcinoma with metastasis to the kidney: report of a case. J Formos Med Assoc 1994; 93: 71 .
8. OHKUMA S, OGASAWARA T, KAWAMURA H.: Right renal metastasis in a patient with hepatocellular carcinoma. Nippon Shokakibyo Gakkai Zasshi 1978; 75: 746 .

9. FUKUSHIMA M, ISOYAMA E, SAKARIDANI N.: Renal metastasis originating from liver cancer. Nipon Hinyokika Gakkai Zasshi 1996; 87: 710.

10. GATTUSO P, RAMZY I, TRUONG L D et al.: Utilization of fine-needle aspiration in the diagnosis of metastatic tumors to the kidney. Diagn Cytopathol 1999; 21: 35.

11. VAN DER POEL HG, ROUKEMA JA, HORENBLAS S, VAN GEEL AN, DEBRUYNE FM.: Metastasectomy in renal cell carcinoma: a multicenter retrospective analisis. European Urology 1999; 35: 197.

Dr. E. Sanz Mayayo

C/ General Margallo, 24 - Esc. I, $2^{\circ} \mathrm{A}$

28020 Madrid

(Trabajo recibido el 13 mayo de 2002) 